\title{
LOS JUEGOS DE ROL COMO ESTRATEGIA DE ENSEÑANZA- APRENDIZAJE PARA FOMENTAR LA ADQUISICIÓN DE COMPETENCIAS: UNA EXPERIENCIA EN LA TITULACIÓN DE GRADO EN TURISMO ${ }^{1}$
}

\author{
LAURA ZUMAQUERO GIL \\ Profesora Contratada-Doctora de Derecho Civil \\ laurazg@uma.es \\ Universidad de Málaga
}

\begin{abstract}
Resumen:
En este trabajo se expone la experiencia llevada a cabo a través de la técnica del juego de rol en una asignatura de introducción al derecho de la titulación de Grado en Turismo. Esta herramienta ha sido utilizada dentro de la modalidad de enseñanza «clases prácticas», con el objetivo de aumentar la motivación de los alumnos, así como fomentar la adquisición de algunas de las competencias que aparecen en la guía de la asignatura y que deben ser sometidas a evaluación. El trabajo recoge también el modelo de rúbrica de evaluación utilizado, además de la valoración que tanto profesor como alumnos realizan de la actividad desarrollada.
\end{abstract}

Palabras clave: Experiencia de innovación docente, clases prácticas, juego de rol, motivación, adquisición de competencias, rúbrica de evaluación.

\begin{abstract}
:
This paper exposes the experience carried out through the technique of role play in a subject of introduction to the civil law of the Degree in Tourism. This tool has been used within the teaching modality "practical classes», in order to increase the motivation of students, as well as encourage the acquisition of some of the skills that appear in the guide of the subject and that must be submitted to evaluation. The work also includes the rubric model that has been used to evaluate the acquisition of such skills, as well as the assessment that both the teacher and students make of the activity carried out.
\end{abstract}

Keywords: Teaching innovation experience, practical classes, role playing, motivation, acquisition of competencies, evaluation rubric.

Sumario: 1. Introducción. 2. Descripción de la experiencia. 2.1. Contextualización. 2.2. Objetivos. 2.3. Metodología. 3. Procedimiento de evaluación y resultados. 3.1. Sistema de evaluación. 3.2. Resultados obtenidos. 4. Valoraciones de profesor y alumnos. 4.1. Valoración del alumno. 4.2. Valoración del profesor. 5. Conclusiones. Bibliografía. Anexo.

\footnotetext{
${ }^{1}$ Este trabajo ha sido realizado en el marco de los Proyectos de Investigación "La simulación como herramienta de enseñanza-aprendizaje práctico en el ámbito jurídico (PIE 17-112)" y “Aprendizaje colaborativo, método del caso y role-playing como metodología para la adquisición de competencias profesionales necesarias en el ámbito jurídico en asignaturas de Grado y Master: el trabajo en equipo (PIE 15-105)”, cuya dirección corre a cargo de la Dra. Carmen Sánchez Hernández.

REJIE Nueva época: Revista Jurídica de Investigación e Innovación Educativa Núm.18, Junio 2018, pp. 43-56 [En línea] http://www.revistas.uma.es/index.php/rejie
} 


\section{Introducción.}

Cuando se trata de reflexionar acerca del modelo de enseñanza más adecuado para un determinado grupo de estudiantes, se debe partir de la idea que no todos los modelos de enseñanza permiten alcanzar los mismos objetivos. Existen distintas maneras de organizar la enseñanza, dependiendo de los propósitos que el profesor se haya marcado en su asignatura. Como afirma Mario De Miguel no es lo mismo que el propósito del profesor sea simplemente transmitir conocimientos al alumno, convirtiéndose en un mero reproductor de conceptos, que enseñarle a aplicar tales conocimientos o promover la participación, el debate y el intercambio entre ellos ${ }^{2}$. Por tanto, habrá que fijar en un primer momento cuáles son los objetivos que queremos alcanzar en nuestra asignatura, a efectos de poder seleccionar la metodología que mejor se adecúe a tales objetivos.

Si entre los objetivos marcados se encuentran la participación activa del alumno, el trabajo colaborativo y la puesta en práctica de los conocimientos adquiridos previamente durante las clases magistrales, la herramienta de los juegos de rol, dentro de un modelo de enseñanza basado en clases prácticas, se presenta de gran utilidad para su consecución. Los juegos de rol permiten involucrar a los alumnos en su propio proceso de aprendizaje a partir de situaciones reales o de contextos simulados, que van a repercutir en el desarrollo de numerosas competencias, imprescindibles en su proceso de formación ${ }^{3}$. Se trata de una herramienta que permite a los alumnos trabajar activamente y de manera cooperativa en el estudio, análisis, desarrollo y resolución de $\operatorname{casos}^{4}$. Aunque la simulación no permite reproducir de forma completa las circunstancias de un caso real, sí sirve para aproximarse a él de una manera bastante fidedigna, resultando un buen sistema para facilitar un aprendizaje orientado a la comprensión y la utilización ${ }^{5}$. Esta metodología basada en principios de trabajo colaborativo puede ser un eficaz complemento educativo para mejorar el interés en los contenidos de la asignatura ${ }^{6}$, además de ser una técnica que permite combinar el trabajo grupal con la toma de decisiones ${ }^{7}$. Los beneficios que aporta a los estudiantes son múltiples. Además de aumentar la motivación y fomentar el trabajo en grupo, permite la reflexión sobre la materia expuesta previamente en clase, genera debate entre los propios miembros del grupo, mejora la capacidad individual de argumentación y de análisis, y permite conseguir un mayor compromiso por parte de los alumnos en cuanto

\footnotetext{
${ }^{2}$ DE MIGUEL DÍAZ, M. (2005), Modalidades de enseñanza centradas en el desarrollo de competencias. Orientaciones para promover el cambio metodológico en el Espacio Europeo de Educación Superior, Universidad de Oviedo, pág. 50.

${ }^{3}$ Para Schutzemberger el role playing es "un instrumento de aprendizaje y perfeccionamiento de las relaciones humanas, una iniciación a la solución de conflictos ulteriores por la solución de conflictos ficticios y la representación de diferentes roles, generalmente tradicionales, familiares, profesionales...". SCHUTZEMBERGER, A. (1979), Introducción al role-playing. El sicodrama, el psicodrama y sus aplicaciones en asistencia social, en las empresas, en la educación y en psicoterapia, $1^{\mathrm{a}}$ ed. Madrid, Marova, págs. 78 - 79.

${ }^{4}$ JOHNSON, R. y SMITH, K. (1998), Active learning: cooperation in the college classroom, Edina, MI, Interaction book company, págs. 21-23.

${ }^{5}$ ORTIZ DE URBINA CRIADO, M., MEDINA SALGADO, S., DE LA CALLE DURÁN, C (2010), "Herramientas para el aprendizaje colaborativo: una aplicación práctica del juego de rol", Teoría de la Educación y Cultura en la Sociedad de la Información, núm. 11(3), pág. 284.

${ }^{6}$ PUIG, H., SAYOS, R., SOLÉ, M (2010), Ejes para la mejora docente en la Universidad, Barcelona, Parcerisa, pág. 74.

${ }^{7}$ RIVAS, M. (2000). Innovación educativa: teoría, procesos y estrategias, Madrid, Síntesis, pág. 290.
} 
a la asistencia a clase ${ }^{8}$. De hecho, la asignación de roles puede ayudar tanto a la delimitación de las tareas comunes que deben desarrollar los miembros del grupo como a la especificación de la responsabilidad y contribución individual de cada uno de los participantes, lo que se puede traducir a su vez en un aumento de los recursos de que disponen los alumnos para regular y hacer progresar la actividad ${ }^{9}$. Todo ello les permite construir su propio conocimiento ${ }^{10}$.

En el campo del derecho, donde hemos llevado a cabo nuestra experiencia, esta metodología fortalece la capacidad de análisis y razonamiento jurídico, fomentando la expresión oral y escrita ${ }^{11}$. Por otra parte, permite mejorar el proceso de aprendizaje de contenidos complejos del derecho ${ }^{12}$. La utilidad de los juegos de rol para el desarrollo de éstas y otras competencias se pone de manifiesto en la experiencia que hemos llevado a cabo durante el curso académico 2016/2017, en una asignatura de introducción al derecho, integrada en el plan de estudios de la titulación de Grado en Turismo. En este trabajo se explica el diseño y puesta en marcha de un sistema de prácticas, que incluye la técnica de los juegos de rol, con un objetivo prioritario: motivar a los alumnos para que participen de forma activa en su propio proceso de aprendizaje. Motivar a los alumnos desde el principio es clave en asignaturas de contenido jurídico, integradas en titulaciones que no pertenecen a las ciencias jurídicas. Si bien el profesorado trata que los alumnos comprendan desde el principio la importancia de la adquisición de este tipo de formación para un futuro desarrollo de su profesión, sin embargo, ellos lo perciben de otro modo, al considerar que se trata de materias que nada tienen que ver con su titulación. Siendo éste un problema al que nos enfrentamos cada año, si no implementamos métodos de enseñanza que fomenten su participación y les ayude a comprobar por ellos mismos el sentido de su proceso de aprendizaje, difícilmente podremos conseguir que adquieran las competencias específicas y genéricas programadas. Junto a este objetivo principal se fijan otros como la mejora de la capacidad de argumentación jurídica, análisis o síntesis o el fomento del trabajo en equipo. En este trabajo se exponen también el sistema de evaluación utilizado

\footnotetext{
${ }^{8}$ Sobre la motivación como una de las fases que integran las fases del proceso que se siguen en el modelo clásico de role playing, MARTIN X. (1992). "El role playing: una técnica para facilitar la empatía y la perspectiva social”, Comunicación, lenguaje y educación, núm. 15, págs. 63 - 64.

9 PLATERO JAIME, M., BENITO HERNÁNDEZ, S., RODRÍGUEZ DUARTE, A. (2012). "Coevaluación y asignación de roles, una experiencia de innovación docente universitaria”, Docencia e Investigación, núm. 22, pág. 12.

${ }^{10}$ Explica Urquidi Martín que la función educativa de este tipo de técnica se entiende desde dos perspectovas diferenciadas: $1^{\circ}$ Desde una perspectiva analítica, al utilizarse para el análisis y la experimentación, permitiendo el aprendizaje sobre el entorno y el fenómeno a estudiar, a partir de la asunción por parte del alumno del rol de experimentador; $2^{\circ}$ Desde una perspectiva deductiva, ya que al implementar simulaciones los alumnos aprenden por las implicaciones o deducciones que obtienen de su participación activa tomando un determinado rol dentro de ésta. Se combinan las carácterísticas propias del juego, pero la situación sobre la que se juega representa un modelo de vida real. URQUIDI MARTÍN, C. (2016), "Simulaciones empresariales para promover el aprendizaje en la educación superior", Aula virtual: contenidos y elementos, Madrid, págs. 664- 665.

${ }^{11}$ SANCHEZ HERNÁNDEZ, C. (2016). "Método del caso y role playing en el ámbito del Derecho Civil”, Presente y futuro de la docencia universitaria, Educación Editora, Orense, pág. 479.

${ }^{12}$ Demuestra este resultado a través de su experiencia, LA SPINA, E. (2011), "Un ejemplo de dinámica de role playing en una asignatura de libre configuración del Derecho”, @ tic Revista de Innovación Educativa, págs. 86-90.
} 
a partir de una rúbrica, los resultados alcanzados, así como las valoraciones que tanto profesor como alumnos realizan de esta actividad.

\section{Descripción de la experiencia.}

\subsection{Contextualización.}

La realización de esta experiencia se enmarca dentro de un conjunto de actividades programadas en una asignatura de primer curso de la titulación de Grado en Turismo de la Universidad de Málaga, denominada «Introducción al Derecho». Esta asignatura forma parte del conjunto de materias de formación básica que integran el plan de estudios. Con ella se pretende que los alumnos adquieran unas nociones generales básicas en materia de derecho civil que les pueden resultar útiles en el ejercicio de su profesión, al permitirles adentrarse en el marco legal de la actividad turística. A partir de las actividades desarrolladas a lo largo del curso, se persigue que los alumnos aprendan a razonar jurídicamente, utilizando conceptos legales prácticos, además de que apliquen la normativa analizada en clase a casos reales propuestos.

La experiencia que hemos llevado a cabo se ha desarrollado en el marco de las clases prácticas de la asignatura. Por clases prácticas entendemos aquella como modalidad organizativa en la que se desarrollan actividades de aplicación de conocimientos a situaciones concretas y de adquisición de habilidades básicas y procedimentales relacionadas con la materia objeto de estudio ${ }^{13}$. En esta actividad han participado 44 alumnos de primer curso, pertenecientes a un grupo de tarde. Este grupo se ha dividido a su vez en dos subgrupos, de 23 y 21 alumnos respectivamente.

\subsection{Objetivos.}

La realización de esta actividad ha tenido por objeto fomentar la adquisición de determinadas competencias que aparecen en la guía de la asignatura, a través de la puesta en práctica de una técnica de enseñanza-aprendizaje que les motivase. En esta ocasión se ha utilizado la herramienta conocida como juego de rol o role playing.

Resulta imprescindible, como paso previo dentro de su proceso de aprendizaje, atraer la atención de los alumnos y aumentar su interés por la asignatura a partir de un sistema activo de enseñanza, que no solamente se centre en el trabajo individual, sino que también incluya el trabajo grupal, de manera que los estudiantes trabajen juntos para lograr objetivos comunes de aprendizaje ${ }^{14}$. Uno de los principales factores de motivación es la necesidad de auto-realización del alumno. Para ello el alumno debe mirar el aprendizaje como un medio, que aunque requiera de esfuerzo y sacrificio, le servirá para alcanzar ciertas satisfacciones, más allá de obtener una calificación. En este proceso los docentes pueden aumentar la motivación de los alumnos asignando notas adecuadas al esfuerzo realizado, además de enriquecer las tareas para que impliquen un reto para el alumno, favorezcan su crecimiento personal y aumenten su capacidad de

\footnotetext{
${ }^{13}$ DE MIGUEL DÍAZ, M., op. cit., pág. 60.

${ }^{14}$ BARKELEY E., CROSS, P. y MAJOR C.H. (2007). Técnicas de aprendizaje colaborativo. Manual para el profesor universitario, Madrid, Morata, pág. 17.
} 
reconocimiento del logro ${ }^{15}$. Junto al aumento de la motivación se han perseguido otros objetivos, generales y específicos, relacionados con la asignatura. Estos objetivos son:

- Fomentar la participación activa en clase y el trabajo en equipo

- Conseguir que aprendan a argumentar jurídicamente el rol asignado

- Aplicación del conocimiento a la práctica y, en concreto, conseguir un aprendizaje significativo en materia de contratación con condiciones generales en el caso en que una de las partes resulte ser un consumidor

- Capacidad de análisis y critica constructiva

- Capacidad de síntesis y plasmación de resultados

\subsection{Metodología.}

El diseño de una metodología adecuada de aprendizaje se presenta fundamental para alcanzar un resultado exitoso en el desarrollo de la experiencia. En este sentido, toda metodología debe componerse de tres elementos fundamentales: modalidades de enseñanza, métodos y estrategias evaluativas ${ }^{16}$. Como hemos indicado anteriormente, nuestra experiencia se ha desarrollado a través de la modalidad de enseñanza clases prácticas, en las que se ha utilizado la herramienta del juego de rol. Esta experiencia se ha desarrollado a lo largo de cinco semanas del segundo cuatrimestre y de manera grupal, a partir de las directrices marcadas por el profesor y que han girado en torno al análisis y resolución de un caso práctico sobre una materia específica del programa.

Se parte de una problemática concreta en el marco de un contrato con condiciones generales de la contratación celebrado con consumidores. Un matrimonio, en calidad de consumidor, compra a través de internet unos billetes de avión, de ida y vuelta, con determinada compañía, para viajar de Málaga a Zurich. El avión sufre un retraso en la hora de salida y, como consecuencia de ello, el matrimonio pierde el vuelo que había contratado con otra empresa para volar a su destino final de vacaciones. Tal retraso, que había sido originado por una situación de overbooking, tuvo como consecuencia que el matrimonio tuviera que pasar dos noches en el destino de conexión antes de poder tomar otro vuelo que les llevase a su destino final; además de tener que comprar ropa y enseres para esta breve estancia en Zurich, tras haberse perdido las maletas y ser éstas entregadas por la compañía al matrimonio dos días más tarde de la fecha de llegada al aeropuerto. A su regreso a Málaga, y tras varias reclamaciones a la empresa sin obtener respuesta alguna, el matrimonio decide demandar a la aerolínea, con la finalidad de obtener una indemnización por los daños y perjuicios sufridos como consecuencia del retraso en el vuelo contratado. Además, solicitan una indemnización que incluya el importe de los gastos que tuvieron que afrontar por la entrega tardía de las maletas, así como por los daños y perjuicios que le fueron ocasionados como consecuencia de la denegación de embarque en el vuelo de regreso a Málaga. La compañía había prohibido el embarque a uno de los miembros de la pareja por presentar el DNI caducado. Sin embargo, el matrimonio pudo volar con otra compañía, por lo que consideran que igualmente podrían haberlo hecho con la compañía con la que inicialmente contrataron.

\footnotetext{
${ }^{15}$ CAL BOUZADA M.I. y VERDUGO MATÉS, M.V. (2016). "El verdadero motor del aprendizaje: la motivación”, Presente y futuro de la docencia universitaria, Orense, pág. 432.

${ }^{16}$ DE MIGUEL DÍAZ, M, op. cit., págs. 18-21.
} 
Por otra parte, solicitan la nulidad de la cláusula que les hacía responsables del pago de los gastos de documentación derivada de la contratación on-line, con el consiguiente efecto restitutorio.

Previamente a la realización de esta actividad se dedicaron dos sesiones, de dos horas de duración, a la institución del arbitraje; y cuatros sesiones, de dos horas de duración, al estudio de las condiciones generales de la contratación.

En la primera fase de esta experiencia los alumnos reciben toda la información sobre la actividad a realizar. Se les informa del concreto problema jurídico que deben abordar y del rol a asumir por cada uno de los grupos. Estos grupos deben estar integrados por un número mínimo de tres alumnos y un máximo de cinco. Cada uno de estos grupos asumirá el rol asignado por el profesor (compañía aérea o matrimonio consumidor). Además se asignará a uno de los grupos el rol de tribunal arbitral, que se encargará de analizar los argumentos aportados por todos los grupos en defensa de su rol, debiendo emitir un veredicto motivado al finalizar la sesión. En esa primera sesión se les facilitó el material con el que poder trabajar: 1. un caso práctico, 2. la legislación aplicable para resolver el caso, 3. jurisprudencia al respecto, 4. artículos doctrinales sobre el tema. Esta primera sesión tuvo lugar tres semanas antes del desarrollo de la actividad de role playing en el aula.

Durante las dos semanas siguientes, los alumnos dedicaron su tiempo a preparar la defensa de su rol, a partir del material aportado. En esta segunda fase de preparación de la actividad todos los grupos deben elaborar una ficha, que será entregada al profesor antes de la sesión de role playing, con los siguientes datos:

\section{$1^{\circ}$ Miembros del grupo \\ $2^{\circ}$ Alumno que hará las veces de portavoz \\ $3^{\circ}$ Distribución de tareas entre sus miembros \\ $4^{\circ}$ Argumentos jurídicos en defensa del rol asignado \\ $5^{\circ}$ Petición que realizan al tribunal arbitral}

El grupo de alumnos que hace las veces de tribunal arbitral debe estudiar todos los argumentos alegados por las partes en conflicto durante la sesión de role playing y analizar su viabilidad, además de emitir un veredicto. El profesor no revisa los argumentos aportados por los alumnos en la ficha hasta la sesión de role playing. En cambio, en el caso del tribunal arbitral, teniendo en cuenta que, al ser un arbitraje de derecho, deben manejar correctamente la legislación y la jurisprudencia aplicable al caso para ofrecer una solución al conflicto que sea sostenible jurídicamente, el trabajo es revisado por el profesor antes de la sesión presencial. Se mantuvieron dos reuniones con cada grupo de alumnos de cara a la preparación de su intervención.

La tercera fase de esta actividad ha consistido en la defensa del rol asignado por los distintos grupos durante la sesión presencial. Teniendo en cuenta que, según el caso propuesto, la demanda fue interpuesta por los consumidores, se dio la palabra, en primer lugar, a los grupos que asumían el rol de consumidor, para que aportasen los argumentos jurídicos en defensa de su postura. Posteriormente, se dio la palabra a los grupos que defendían a la compañía aérea. 
Una vez expuestos por los grupos sus argumentos, el tribunal arbitral, pasa a deliberar, disponiendo de un tiempo de 15 minutos antes de emitir su veredicto. En esta tercera fase el papel del profesor es de mero observador. Es el propio tribunal el que dirige la sesión, organizando el turno de palabra y formulando las preguntas oportunas para poder tomar una decisión al respecto.

En una sesión posterior, que tiene lugar la semana siguiente a la realización del role playing, se analizan los argumentos vertidos en clase para que todos los alumnos tengan claro si han utilizado o no argumentos de calidad para defender su rol y las razones que han llevado a que sus pretensiones fueran estimadas o desestimadas. Tras esta sesión, se les pide que reflexionen acerca de la actividad realizada, debiendo entregar un informe de conclusiones, en el que el grupo haga constar su posición final respecto al asunto, con independencia del rol asignado inicialmente. Deberán explicar los motivos que les han llevado a adoptar esa posición, además de recoger, a modo de síntesis, los argumentos vertidos en clase en defensa de una u otra postura.

\section{Procedimiento de evaluación y resultados.}

\subsection{Sistema de evaluación.}

Para la evaluación de esta actividad se ha utilizado la rúbrica que aparece como anexo al final de este documento, en la que se valora la capacidad de argumentación jurídica, la capacidad de análisis y síntesis, así como la capacidad de plasmar conclusiones y de trabajar en equipo. Cada una de estas competencias va asociada a dos indicadores con cuatro niveles de logro. La competencia relativa a la capacidad de argumentación está asociada a los indicadores «número de argumentos» y «calidad de los mismos». La competencia sobre el trabajo en equipo está asociada a los indicadores «distribución de tareas» y «realización de las actividades programadas». La competencia sobre la capacidad de análisis, síntesis y de plasmar conclusiones está asociada a los indicadores «número de conclusiones» y «análisis y síntesis».

Cada indicador va asociado a distintos niveles de logro con una puntuación determinada. Los niveles de logro se traducen en actuaciones realizadas por el grupo, en relación a cada una de las competencias evaluadas, que permiten al profesor conocer el nivel de éxito de la actuación realizada. La puntuación se distribuye del siguiente modo:

-Nivel de logro 1 equivale a 0 puntos

-Nivel de $\operatorname{logro} 2$, equivale a 1 punto

-Nivel de logro 3 , equivale a 2 puntos

-Nivel de logro 4 , equivale a 3 puntos

Para poder extraer la nota final del grupo, cada intervalo de puntos va asociado a una calificación determinada. Esta calificación puede ser suspenso, aprobado, notable o sobresaliente. Si el grupo obtiene en el desarrollo de la actividad, tras aplicar la rúbrica de evaluación, menos de 9 puntos, su calificación final será de suspenso. Si obtiene una puntuación entre 10 y 12 puntos, la calificación será de aprobado. Si la puntuación oscila entre 13 y 15 puntos, el grupo obtendrá una calificación de notable. Finalmente, 
si la puntuación alcanzada por el grupo está entre los 16 y los 18 puntos, la calificación de la actividad será de sobresaliente.

Esta rúbrica ha servido para evaluar el trabajo grupal y, en ningún caso, el trabajo individual. A lo largo del curso se han realizado otro tipo de actividades que han permitido la evaluación individual del alumno. A través de una metodología tradicional de clases prácticas, en la que se proponía al alumno la resolución de un caso fuera del aula, para su posterior corrección en clase, se ha tratado de fomentar el debate, puesto que son los propios alumnos los que deben ofrecer distintas soluciones al caso. El profesor actúa de moderador durante la fase de debate y solamente al final de la clase interviene tratando de aclarar algunas cuestiones que hayan planteado dudas durante la corrección o para aportar argumentos jurídicos distintos a los propuestos por los propios alumnos.

\subsection{Resultados obtenidos.}

Los resultados obtenidos a partir de esta rúbrica de evaluación han sido muy positivos. De los 11 grupos participantes, 4 han obtenido una calificación de sobresaliente (14 alumnos), 4 de notable (18 alumnos) y 3 de aprobado (12 alumnos).

Del análisis detallado de los resultados alcanzados por los distintos grupos respecto de cada una de las competencias evaluadas se extrae que la puntuación más elevada se alcanza en la competencia relacionada con la capacidad de argumentación jurídica. El 90\% de los grupos utilizan todos los argumentos posibles para la defensa de su rol, siendo todos ellos argumentos de calidad. Solo el $10 \%$ restante aporta algunos argumentos de calidad en defensa de su rol, estando todos ellos fundamentados jurídicamente.

Respecto a la distribución de tareas, 10 de los 11 grupos distribuyen las tareas entre sus miembros y, posteriormente, ponen en común el trabajo realizado. Aunque todos los grupos realizan el trabajo programado, se detecta que uno de los grupos presenta el trabajo gracias a la asunción de todas las tareas por uno solo de sus miembros.

Algo menos exitosa se presenta la evaluación relativa a la última competencia que recoge la tabla. Aunque todos los grupos presentan el informe de conclusiones, solamente dos de ellos realizan la actividad propuesta consistente en recoger a modo de síntesis los argumentos jurídicos utilizados por los distintos grupos para la defensa de su rol.

\section{Valoraciones de profesor y alumnos.}

\subsection{Valoración de los alumnos.}

Los alumnos que participaron en esta experiencia fueron encuestados por el profesor de manera anónima, con objeto de conocer su grado de satisfacción con la actividad realizada. En la encuesta se les preguntaba si la actividad de role playing había satisfecho sus expectativas y si consideraban que los objetivos inicialmente propuestos habían sido alcanzados (mejorar su capacidad de argumentación jurídica, aumento de la 
motivación, mejora de la capacidad de análisis y síntesis, así como de extraer conclusiones). También se les preguntó por la valoración que realizan del trabajo grupal.

El grado de satisfacción de los alumnos con la actividad realizada es bastante bueno. La mayoría de los alumnos encuestados estaban satisfechos con el desarrollo de las clases prácticas a través de la técnica de los juegos de rol, prefiriendo este tipo de actividad que el sistema de las prácticas individuales. 37 de los 40 alumnos encuestados afirmaron que la actividad les había interesado mucho, mostrándose a favor de su continuidad en sucesivas ediciones, al interesarles más que las prácticas individuales. Por lo que respecta a los objetivos perseguidos, llama la atención la divergencia existente entre la valoración que realiza el profesor del aprendizaje de los alumnos y la visión que ellos tienen de sí mismos. Mientras 21 alumnos contestaron que consideraban que habían mejorado mucho su capacidad de argumentación, 18 entendieron que habían mejorado poco y 1 que no había mejorado nada. En cuanto a la valoración que realizan del trabajo en grupo, 30 de los alumnos encuestados respondieron de manera favorable al trabajo colaborativo, mientras que 10 se mostraron contrarios a trabajar en equipo. Entre sus argumentos:

- la división del trabajo entre los miembros del grupo solo te permite aprender una parte del trabajo

- se detecta falta de comunicación en el grupo

- no se toman en cuenta algunas aportaciones de los compañeros

- es difícil ponerse de acuerdo entre varias personas

- poca participación por parte de algún compañero

- todos los compañeros no tiene el mismo nivel

Los alumnos identifican en la encuesta como ventajas e inconvenientes de esta actividad las siguientes:

-Ventajas:

$1^{\circ}$ Permite el trabajo colaborativo y la división de tareas

$2^{\circ} \mathrm{El}$ aprendizaje se hace más ameno

$3^{\circ}$ Fomenta el debate posterior

$4^{\circ}$ Permite trabajar la simulación de casos reales

-Inconvenientes:

$1^{\circ}$ Mayor dificultad en la planificación del trabajo

$2^{\circ}$ Existen opiniones enfrentadas

$3^{\circ}$ Mayor dedicación

$4^{\circ}$ Surgen conflictos entre los compañeros respecto al tiempo de dedicación de cada uno

$5^{\circ}$ Dificulta conocer los errores individuales

\subsection{Valoración del profesor.}

Se puede afirmar en términos generales, a partir de la experiencia realizada, que la técnica del juego de rol ha permitido que los alumnos que han participado de la misma 
vean mejoradas en mayor o menor medida las competencias que han sido objeto de evaluación. Además de motivarles para trabajar en la asignatura. Desde esta perspectiva, la experiencia puede calificarse de muy positiva.

Por lo que respecta a la capacidad de trabajo en equipo, parece conveniente articular un mecanismo de control del efectivo cumplimiento de tareas entre los miembros del grupo a efectos de evitar el rechazo a esta forma de trabajo por parte de algunos alumnos, que prefieren trabajar individualmente antes que de manera grupal. Ello requerirá, en sucesivas experiencias, concertar reuniones con los distintos equipos, para hacer un seguimiento de la distribución de tareas, su cumplimiento y garantizar la puesta en común del trabajo de todos para tomar decisiones por consenso. Ello permitiría a su vez revisar los argumentos utilizados por los grupos para la defensa de su rol, detectando así la existencia de errores jurídicos relevantes.

\section{Conclusiones.}

Tras reflexionar, una vez finalizado el curso, acerca de las fortalezas y debilidades de esta experiencia y la conveniencia o no de continuar con ella en futuras ediciones, concluimos que la misma ha servido para alcanzar en mayor o menor medida los objetivos marcados, presentando fortalezas y oportunidades que hacen de la técnica de los juegos de rol una herramienta útil para el trabajo práctico de los alumnos que cursan una asignatura introductoria al derecho, en una titulación, como es la de turismo, que no pertenece a las ciencias jurídicas.

Entre sus fortalezas detectamos que el uso de esta técnica aumenta la motivación de los alumnos, hace más ameno su aprendizaje y también lo mejora. Entre las oportunidades que brinda esta herramienta se encuentran, el trabajo en equipo, la obligación de los alumnos de conciliar posturas discrepantes y el aumento de la capacidad de negociación.

Por lo que respecta a sus debilidades, entendemos que la forma de desarrollar esta experiencia plantea problemas respecto al trabajo grupal y su evaluación. Resulta imprescindible revisar el trabajo de todos los grupos y no solamente de aquéllos que asumen el rol de tribunal arbitral. Ello va a permitir que las intervenciones sean más correctas desde el punto de vista argumentativo, ya que las incorrecciones jurídicas son detectadas con anterioridad a la sesión presencial, además de poder realizar un seguimiento real del trabajo que cada uno de sus miembros está realizando. De no hacerse este seguimiento, no es posible comprobar el cumplimiento verdadero de la distribución de tareas entre los miembros, puesto que partimos simplemente de las manifestaciones realizadas por cada uno de ellos.

Se ha detectado también la necesidad de aumentar el tiempo de duración de la sesión presencial de 1 hora a 1 hora y 30 minutos de duración. La idea es que todos los grupos dispongan de más tiempo para su exposición y que el tribunal arbitral pueda tener más tiempo para deliberar y posteriormente emitir un veredicto motivado. 


\section{Bibliografía.}

-BARKLEY E., CROSS P. Y MAJOR C.H. (2007). Técnicas de aprendizaje colaborativo. Manual para el profesor universitario, Madrid, Morata, 2007.

-CAL BOUZADA M.I. y VERDUGO MATÉS, M.V. (2016). "El verdadero motor del aprendizaje: la motivación", Presente y futuro de la docencia universitaria, Orense, págs. 431-435.

-DE MIGUEL DÍAZ M. (2005). Modalidades de enseñanza centradas en el desarrollo de competencias. Orientaciones para promover el cambio metodológico en el Espacio Europeo de Educación Superior. Universidad de Oviedo.

-JOHNSON R. Y SMITH K. (1998). Active learning: cooperation in the college classroom, Edina, MI, Interaction book company.

-LA SPINA, E. (2011). "Un ejemplo de dinámica de role playing en una asignatura de libre configuración del Derecho”, @ tic Revista de Innovación Educativa, págs. 86-90.

-LÓPEZ F. (2005). Metodología participativa en la enseñanza universitaria, Narcea Ediciones, Madrid.

-MARTIN X. (1992), "El role playing: una técnica para facilitar la empatía y la perspectiva social”, Comunicación, lenguaje y educación, núm. 15, págs. 63-67.

-ORTIZ DE URBINA M., MEDINA S., DE LA CALLE C. (2010). "Herramientas para el aprendizaje colaborativo: una aplicación práctica del juego de rol". Teoría de la Educación. Educación y Cultura en la Sociedad de la Información, TESI, 11(3), pags. 277-300.

-PLATERO JAIME, M., BENITO HERNÁNDEZ, S., RODRÍGUEZ DUARTE, A. (2012). "Co-evaluación y asignación de roles, una experiencia de innovación docente universitaria”, Docencia e Investigación, núm. 22, págs. 7-29.

-PUIG H., SAYOS R., SOLÉ, M (2010). Ejes para la mejora docente en la universidad, Barcelona, Octaedro.

-RIVAS M. (2000). Innovación educativa: teoría, procesos y estrategias, Madrid, Síntesis.

-SÁNCHEZ HERNÁNDEZ C. (2016). Método del caso y role playing en el ámbito del Derecho Civil. Presente y futuro de la docencia universitaria, Educación Editora, Orense, págs. 477-481.

-SCHUTZEMBERGER A. (1979). Introducción al role-playing. El sicodrama, el psicodrama y sus aplicaciones en asistencia social, en las empresas, en la educación y en psicoterapia, Madrid, Marova. 
-URDIQUI MARTIN, C. (2016) "Simulaciones empresariales para promover el aprendizaje en la educación superior", Aula virtual: contenidos y elementos, MacGraw Hill, Madrid, págs. 663 - 677.

Anexo.

\begin{tabular}{|c|c|c|}
\hline $\begin{array}{c}\text { COMPETENCIAS A } \\
\text { EVALUAR }\end{array}$ & INDICADORES & NIVELES DE LOGRO \\
\hline Capacidad de argumentación & $\begin{array}{l}\text { 1. Número de argumentos } \\
\text { jurídicos } \\
------------ \\
--- \\
\text { 2. Calidad de los mismos }\end{array}$ & $\begin{array}{l}\text { N1. El grupo no aporta } \\
\text { argumentos jurídicos para la } \\
\text { defensa de su rol } \\
\text { N2. El grupo aporta algún } \\
\text { argumento jurídico para la } \\
\text { defensa de su rol } \\
\text { N3. El grupo aporta varios } \\
\text { argumentos jurídicos para la } \\
\text { defensa de su rol } \\
\text { N4. El grupo utiliza todos los } \\
\text { argumentos jurídicos posibles } \\
\text { para la defensa de su rol } \\
----------- \\
--- \\
\text { N1. El grupo no aporta ningún } \\
\text { argumento de calidad } \\
\text { N2. El grupo aporta algunos } \\
\text { argumentos de calidad, pero no } \\
\text { los fundamenta en la } \\
\text { legislación aplicable al caso } \\
\text { N3. El grupo aporta algunos } \\
\text { argumentos jurídicos de } \\
\text { calidad y los fundamenta con la } \\
\text { legislación aplicable al caso } \\
\text { N4. Todos los argumentos } \\
\text { aportados por el grupo son de } \\
\text { calidad, además de estar } \\
\text { fundamentados jurídicamente }\end{array}$ \\
\hline
\end{tabular}




\begin{tabular}{|c|c|c|}
\hline $\begin{array}{c}\text { COMPETENCIAS A } \\
\text { EVALUAR }\end{array}$ & INDICADORES & NIVELES DE LOGRO \\
\hline & $\begin{array}{l}\text { 1. Distribución de tareas } \\
------------ \\
-- \\
\text { 2. Realización de las } \\
\text { actividades programadas }\end{array}$ & $\begin{array}{l}\text { N1. El grupo no realiza } \\
\text { ninguna distribución de tareas } \\
\mathrm{N} 2 \text {. El grupo distribuye } \\
\text { distintas tareas entre sus } \\
\text { miembros por igual, pero no } \\
\text { las ponen en común } \\
\mathrm{N} 3 \text {. El grupo divide las tareas } \\
\text { entre sus miembros, las ponen } \\
\text { en común, pero no eligen por } \\
\text { consenso los argumentos } \\
\text { definitivos } \\
\mathrm{N} 4 \text {. El grupo divide las tareas } \\
\text { entre sus miembros, las ponen } \\
\text { en común, y eligen por } \\
\text { consenso los argumentos que } \\
\text { van a utilizar } \\
------------ \\
-- \\
\mathrm{N} 1 \text {. El grupo no realiza el } \\
\text { trabajo programado } \\
\mathrm{N} 2 \text {. El grupo realiza la primera } \\
\text { parte del trabajo, pero no la } \\
\text { segunda } \\
\mathrm{N} 3 \text {. El grupo realiza parte del } \\
\text { trabajo encargado, pero alguna } \\
\text { actividad se entrega fuera de } \\
\text { plazo } \\
\mathrm{N} 4 \text {. El grupo realiza todo el } \\
\text { trabajo encargado en el plazo } \\
\text { fijado }\end{array}$ \\
\hline $\begin{array}{l}\text { Capacidad de análisis y } \\
\text { síntesis, así como de plasmar } \\
\text { conclusiones }\end{array}$ & $\begin{array}{l} \\
------------ \\
---\end{array}$ & 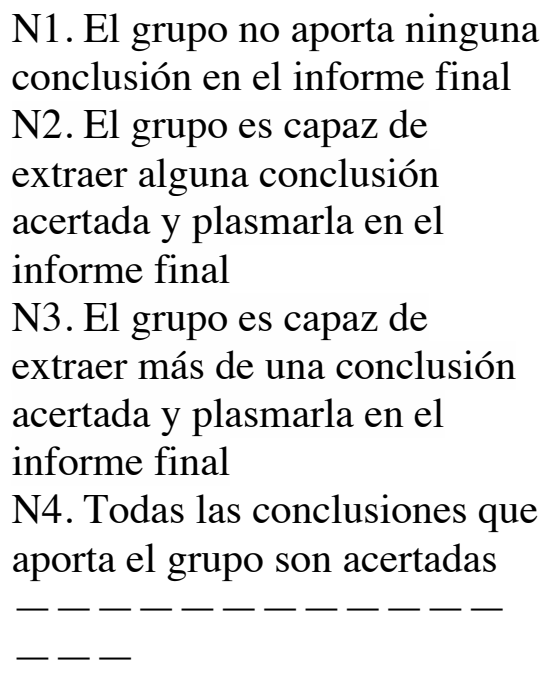 \\
\hline
\end{tabular}




\begin{tabular}{|l|l|l|}
\hline $\begin{array}{c}\text { COMPETENCIAS A } \\
\text { EVALUAR }\end{array}$ & \multicolumn{1}{|c|}{ INDICADORES } & \multicolumn{1}{|c|}{ NIVELES DE LOGRO } \\
\hline 2. Análisis y síntesis & $\begin{array}{l}\text { N1. El grupo no aporta ninguna } \\
\text { síntesis de los argumentos } \\
\text { vertidos en clase } \\
\text { N2. El grupo aporta una } \\
\text { síntesis incompleta de los } \\
\text { argumentos vertidos en clase } \\
\text { N3. El grupo aporta una } \\
\text { síntesis solamente de los } \\
\text { argumentos que apoyan la } \\
\text { defensa de un rol } \\
\text { N4. El grupo sintetiza } \\
\text { perfectamente todos los } \\
\text { argumentos vertidos en clase } \\
\text { en defensa de ambos roles }\end{array}$ \\
\hline
\end{tabular}

Tabla 1.-Rúbrica de evaluación 